\title{
Feminizing genitoplasty in childhood: aiming for achievable outcomes
}

\author{
Amr Abdelhamid AbouZeid(B)
}

\begin{abstract}
Background: Early genital reconstruction may be recommended in cases of congenital adrenal hyperplasia to avoid the stigma that can affect these individuals with variable degrees through different cultures. However, the separation and mobilization of a high vagina has remained challenging with less satisfactory surgical outcomes. Therefore, the value of early vaginal reconstruction has been questioned in favour of delaying the whole repair after puberty. In this report, the author has adopted the third option in-between, which comprises early reconstruction of the external genitalia and delaying the more challenging vaginal reconstruction (if needed) to be performed after puberty.

Results: The study included ten consecutive cases of CAH who underwent feminizing genitoplasty during the period 2016 through 2019. Their age at operation ranged from 8 to 84 months (mean 31; median 15).

In five cases (50\%), the technique of limited urogenital sinus mobilization adopted in this report succeeded in bringing the vaginal introitus down to the perineum. Those cases had originally a low vagina. In the rest of cases, labial retraction showed a common but wide urogenital introitus perfectly lined by urogenital mucosa. The outcomes have been considered satisfactory to a great extent for both parents and doctors regarding cosmesis and lack of functional complications (voiding problems). Longer follow up is still needed to assess the sexual function when these girls become sexually active.

Conclusion: In cases of congenital adrenal hyperplasia, reduction clitoroplasty combined with partial urogenital sinus mobilization can achieve predictable and satisfactory outcomes. In about 50\% of cases, this approach is sufficient to bring the vagina down to the perineum. In the other half of cases with higher vagina, the possibility of satisfactory sinus intercourse can be studied when these girls grow and become sexually active; otherwise, a delayed vaginal reconstruction may turn to be necessary.
\end{abstract}

Keywords: Congenital adrenal hyperplasia, Clitoris, Vestibuloplasty, Urogenital sinus, Genitalia

\section{Background}

Congenital adrenal hyperplasia $(\mathrm{CAH})$ represents the most common diagnosis among cases presenting with genital ambiguity $[1,2]$. Affected females $(46 \mathrm{XX}$, a normal female sex chromosomes) should receive appropriate hormonal replacement therapy (corticosteroids \pm mineralocorticoids) and can be expected to have normal fertility [3]. Except for cases with severe virilization and

\footnotetext{
Correspondence: amrabdelhamid@hotmail.com
}

Pediatric Surgery Department, Faculty of medicine, Ain Shams University,

Lotefy El-Sayed Street, 9 Ain Shams University Buildings, Abbassia, Cairo

11657, Egypt

(c) The Author(s). 2020 Open Access This article is licensed under a Creative Commons Attribution 4.0 International License, which permits use, sharing, adaptation, distribution and reproduction in any medium or format, as long as you give appropriate credit to the original author(s) and the source, provide a link to the Creative Commons licence, and indicate if changes were made. The images or other third party material in this article are included in the article's Creative Commons licence, unless indicated otherwise in a credit line to the material. If material is not included in the article's Creative Commons licence and your intended use is not permitted by statutory regulation or exceeds the permitted use, you will need to obtain permission directly from the copyright holder. To view a copy of this licence, visit http://creativecommons.org/licenses/by/4.0/. 
management of cases of $\mathrm{CAH}$, and the rationale behind this approach.

\section{Methods}

The study included girls with genital ambiguity (46 XX, a normal female sex chromosomes) diagnosed as CAH. Cases were referred from the paediatric endocrinology unit that represents the mainstay for treatment and follow up of these patients. Preoperative evaluation included contrast studies (genitograms), endoscopy and more recently, non-contrast pelvic MRI studies. In a previous study [5], we have demonstrated that in most cases of $\mathrm{CAH}$ (about 95\%), the level of urogenital confluence (vaginal take off from the urethra) can be related to the distal end of pubic symphysis. The confluence is considered low (50\%) if it reaches below the distal end of the pubic symphysis when the vagina is located in the superficial perineal pouch (type 1); otherwise, the vagina will be located higher in the pelvis (type2). Occasionally (5\%), the urogenital confluence may be located exceptionally high opposite the level of mid pubis (type $2 \mathrm{~b}$ ) with higher vaginal termination in the upper pelvis [5].

At their consensus meeting [4], experts from all over the world came to three surgical options for cases of CAH: (1) vaginal connection to the perineum with clitoral reduction, (2) clitoral reduction without vaginal surgery and (3) no or delayed surgery. At our region, the third option may not be so appealing (either for parents or medical community) especially when the decision on choosing the female gender is straightforward as in most of cases of CAH. Here, we are left with two options. Owing to the reported less satisfactory outcomes with the first option, the author has adopted the second one.
Based on preoperative evaluation, parents of cases with a low vagina were informed about the procedure as a single stage full correction. For those with a high vagina, parents were informed about the value of operation to correct the external genitalia and that reassessment at puberty is necessary for the possibility of a delayed vaginoplasty. The other option of single-stage reconstruction (TUM, ASTRA, laparoscopic approach) for a high vagina was also discussed with the parents when they will need to shift to another consultant.

In this case series, the same surgical principles were applied for all cases whatever the level of the urogenital confluence delaying vaginal reconstruction if needed to be performed later (after puberty).

\section{Skin incision (Fig. 1)}

We start by degloving of the virilized clitoris exactly as in hypospadias surgery taking care to keep a collar of foreskin around the glans. To complete the exposure of deeper components of the virilized clitoris, an extension is made via a midline skin incision through the perineum starting at the common urogenital orifice and extending backwards to the predestined site of the vaginal orifice (below and behind the lower end of the pubis). Careful dissection of the perineal skin from the underlying erectile tissue analogous to the bulb of corpus spongiosum is made. Inadvertent traversing the erectile tissue during dissection would lead to bleeding obscuring the operative field (bipolar diathermy can be very helpful during this step).

Separation (mobilization) of the urogenital sinus (Fig. 2) A well-applied self-retaining retractor (lone star retractor; mastoid retractor) greatly improves the surgical

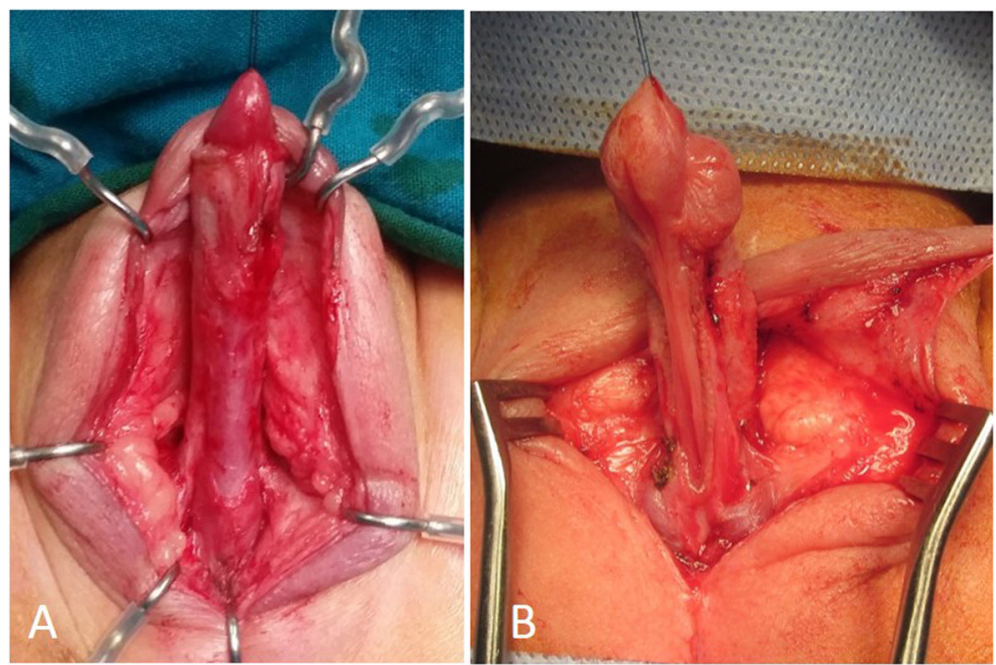

Fig. 1 Degloving of the genital tubercle as a first step of feminizing genitoplasty in two different cases of $\mathrm{CAH}(\mathbf{a}, \mathbf{b})$. Their age at operation was 8 months and 10 months, respectively 

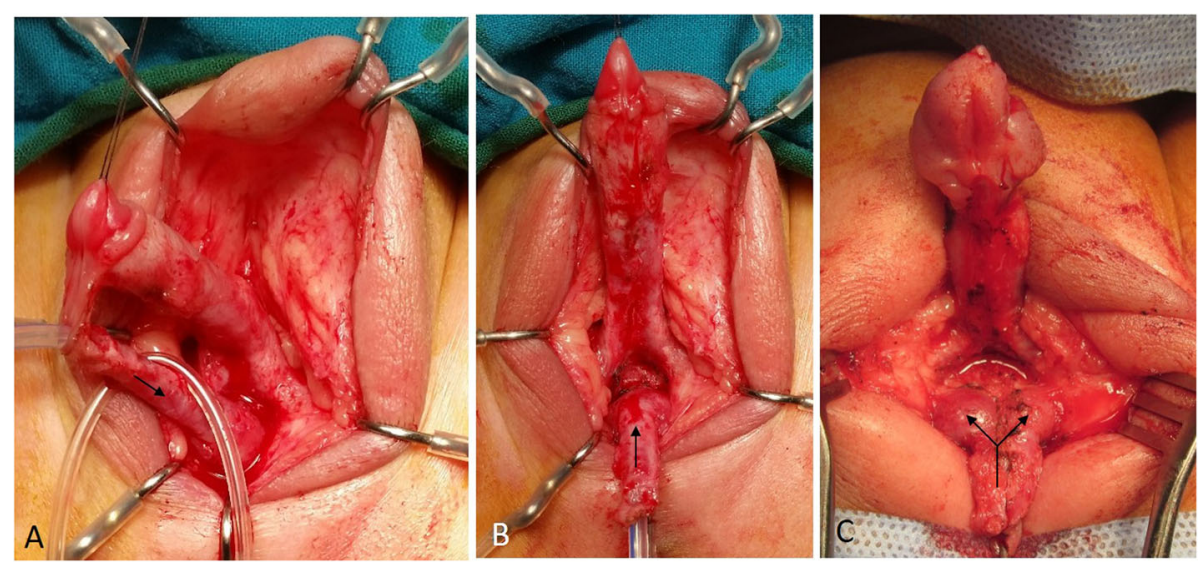

Fig. 2 Feminizing genitoplasty (cont.). Separation of the urogenital sinus (arrow) together with the surrounding ventral erectile tissue from the dorsal corporeal bodies of the genital tubercle. a, b First case 8-month-old girl with CAH. c Second case 10-month-old girl with CAH. Note the divergence of the bulbus spongiosum in $\mathbf{c}$ compared to that in $\mathbf{b}$

exposure. Dissection is continued on the dorsal aspect of the virilized clitoris down to its origin from the under surface of the pubis; this is marked by the bifurcation of the two dorsal corporeal bodies (corpora cavernosa). The common urogenital sinus (some authors would prefer calling it distal urethra) [4] together with its surrounding ventral erectile tissue (analogous to corpus spongiosum) are separated from the dorsal corporeal bodies. It is the author preference to always perform partial mobilization (stopping at the lower end of the pubis) and avoid extending the dissection into the upper retropubic space that may distort the normal anatomy of the bladder neck (total mobilization). In a previous study, we have demonstrated a new sign in MRI which is the divergence of the bulbus spongiosum indicating for a low vaginal descent (below the distal end of pubic symphysis) [5, 6]. The bulb divergence was also recognized intraoperatively to be associated with a low vagina (note the difference between Fig. $2 b$ and c).

\section{Reduction clitoroplasty (Figs. 3, 4 and 5)}

A rubber tourniquet is applied at the base of each of the dorsal corporeal bodies to control bleeding during surgical dissection (Fig. 3). Two longitudinal incisions are made in the deep fascia at 3 and 9 o'clock extending along the length of the corporeal bodies to safely separate the dorsal neurovascular bundle from the underlying erectile tissue (Fig. 4). The corporeal bodies are ligated at their base just distal to the tourniquet excluding the dissected neurovascular bundle from the ligation. The
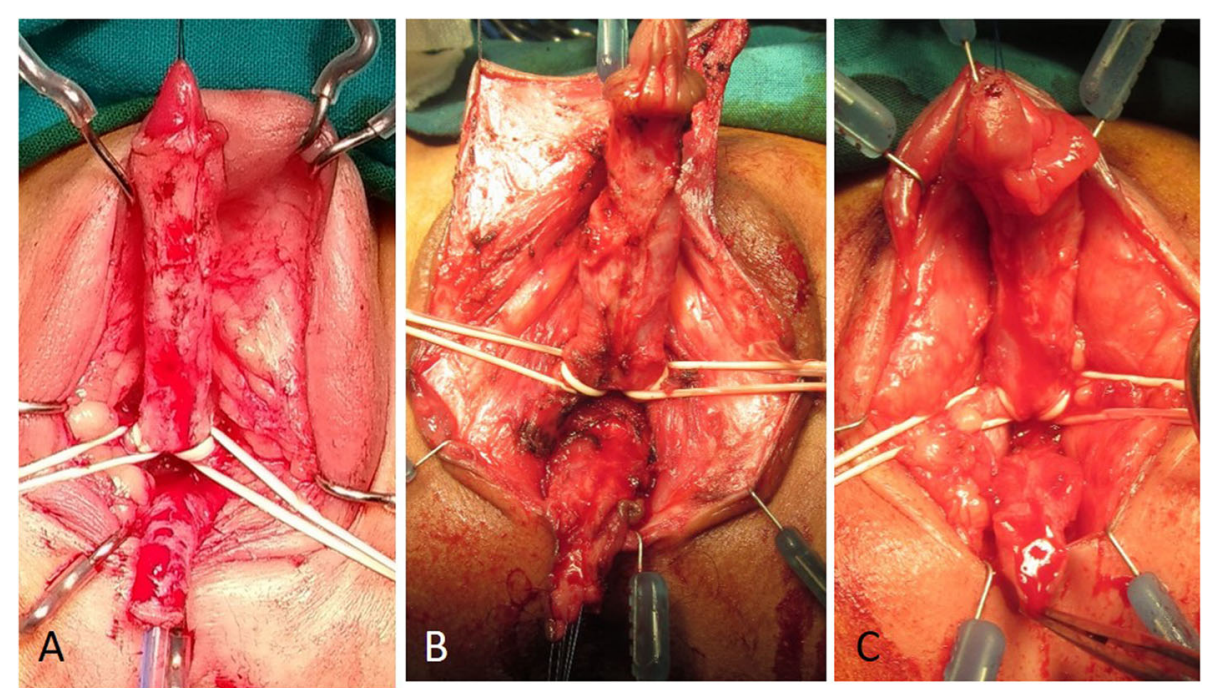

Fig. 3 Feminizing genitoplasty (cont.). a-c Three different cases of CAH (46, XX, a normal female sex chromosomes); their age was 8 months, 84 months and 15 months, respectively. A rubber tourniquet is applied at the base of each of the dorsal corporeal bodies to control bleeding during surgical dissection 

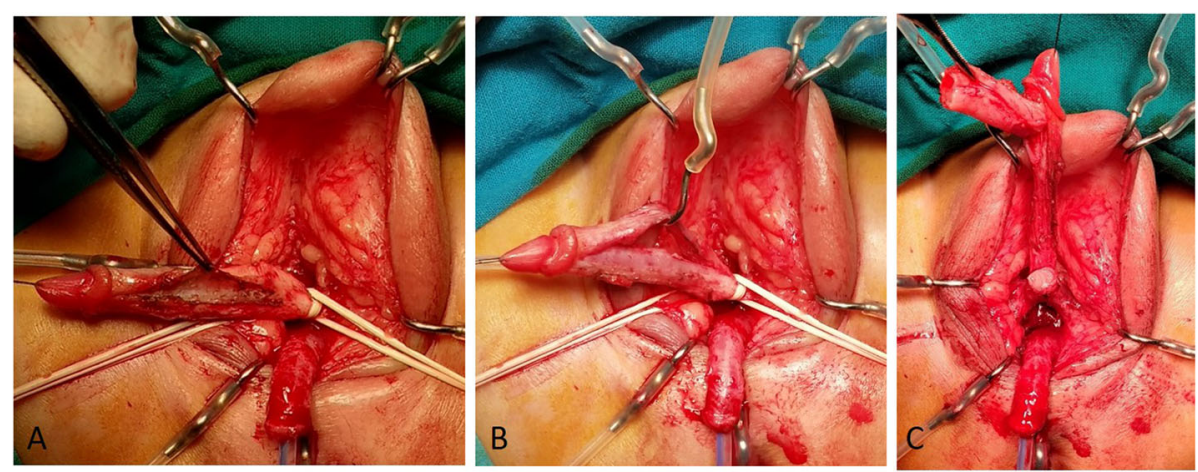

Fig. 4 Feminizing genitoplasty (cont.). An 8-month-old girl with CAH. a, b Dissection of the dorsal neurovascular bundle. c The hypertrophied dorsal corporeal bodies are excised starting distal to the ligature up to the glans

rubber tourniquets are then removed after securing the base of the corporeal bodies by suture ligation. The virilized dorsal corporeal bodies are excised starting distal to the ligature up to the glans; the latter is preserved in continuity with the dorsal neurovascular bundle (Fig. 5). Reduction in the size of a large glans can be performed at this stage. The glans is then re-sutured to the ligated corporeal stump. Sometimes, it is difficult to judge on the size of the glans whether it needs further reduction or not. In such a situation, the author would prefer to wait after healing and reassess the size of the glans. Glanular reduction (if needed) can be performed at a delayed complementary stage 6 months later.

\section{Vestibuloplasty (Figs. 6 and 7)}

A classic 'lay-open' incision is made through the ventral wall of the dissected urogenital sinus. Again, the bipolar diathermy is helpful in dividing the erectile tissue on the ventral aspect of the sinus. This step is important to split the ventral erectile tissue (analogous to bulb of corpus spongiosum) from the midline displacing it laterally on both sides of the new introitus (Fig. 6) and to expose the vaginal orifice in cases with a low vagina (Fig. 7). In the latter, the well-exposed posterior vaginal wall can now be sutured either directly to the perineal skin or indirectly to a previously prepared posterior 'Fortinoff' skin flap. In cases with higher vaginal take-off, the posterior wall of the split-open common urogenital sinus can be similarly sutured to the perineal skin at this stage, after dividing and displacing the ventral erectile tissue laterally on both sides of the new introitus (Fig. 6).

\section{Fixation of the urethra to the clitoris (Figs. 8 and 9)}

Another midline incision is made dorsally through the dissected urogenital sinus splitting it into two equal flaps that will be rotated and sutured to the mucosal collar on both sides encircling the glans. This step has been shown to improve cosmesis by providing urogenital mucous membrane lining for the new vestibule. The dorsal skin of the virilized clitoris is incised in the midline (Byars' flaps fashion) taking care to keep enough dorsal skin for covering the glans (hood), while the Byars' flaps are used to reconstruct the labia minora on both sides of the vestibule.

\section{Follow up}

Special attention was given to exclude possible postoperative atrophy of the glans and voiding problems. Beside the regular follow up at the paediatric endocrinology clinic, operated cases were instructed to keep on regular
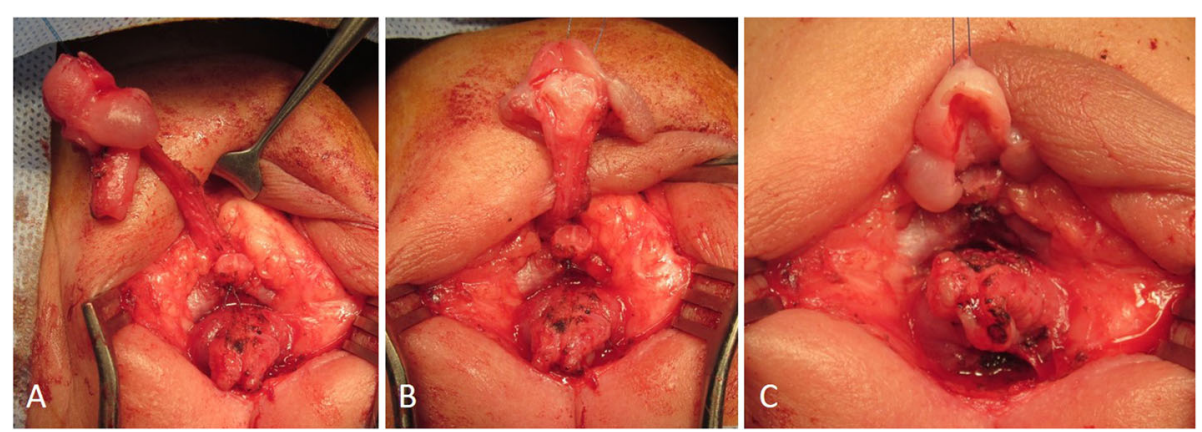

Fig. 5 Feminizing genitoplasty (cont.). A 10-month-old girl with CAH. a, b The glans is preserved in continuity with the dissected dorsal neurovascular bundle. $\mathbf{c}$ The glans is then re-sutured to the ligated corporeal stump 

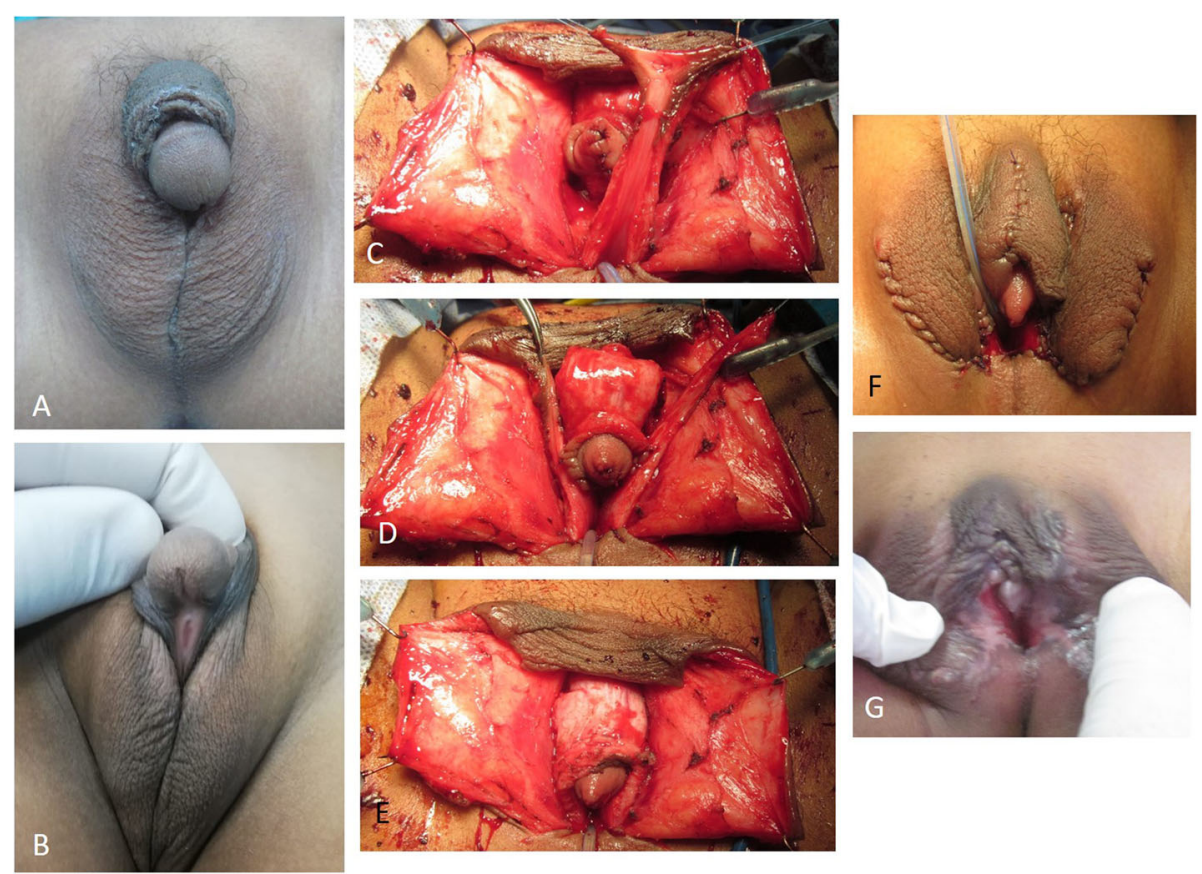

Fig. 6 Feminizing genitoplasty (cont.). A 59-month-old girl with CAH. a, b Preoperative virilized appearance of external genitalia. c A classic 'layopen' incision is made through the ventral wall of the dissected urogenital sinus. $\mathbf{d}$, e Another midline incision is made dorsally through the dissected urogenital sinus splitting it into two equal flaps that will be rotated and sutured to the mucosal collar on both sides encircling the glans. $\mathbf{f}, \mathbf{g}$ The appearance of external genitalia immediately postoperatively and after 1 month, respectively. Note the wide vestibule perfectly lined by urogenital mucosa in $\mathbf{g}$

visits (at wider intervals, 6-12 months) at our surgical clinic for reassessment until and through puberty. This is important to reassess the repaired introitus and correct any obstacles before planning for marriage.

\section{Results}

The study included ten consecutive cases of $\mathrm{CAH}$ who underwent feminizing genitoplasty during the period 2016 through 2019. Their age at operation ranged from 8 to 84 months (mean 31; median 15). Postoperative oedema was a common but transient complication.
Wound disruption occurred in a single case that required delayed secondary suturing.

Follow up period ranged from 6 months to 3 years. All cases had intact glans and normal micturition. In three cases, the parents showed persistent major concern towards the large size of the retained glans, and a complementary second stage was performed under general anaesthesia to reduce its size as requested (Fig. 10). This represented a simple procedure with minimal and superficial dissection compared to the primary operation. The sensitivity of the glans was unintentionally confirmed at

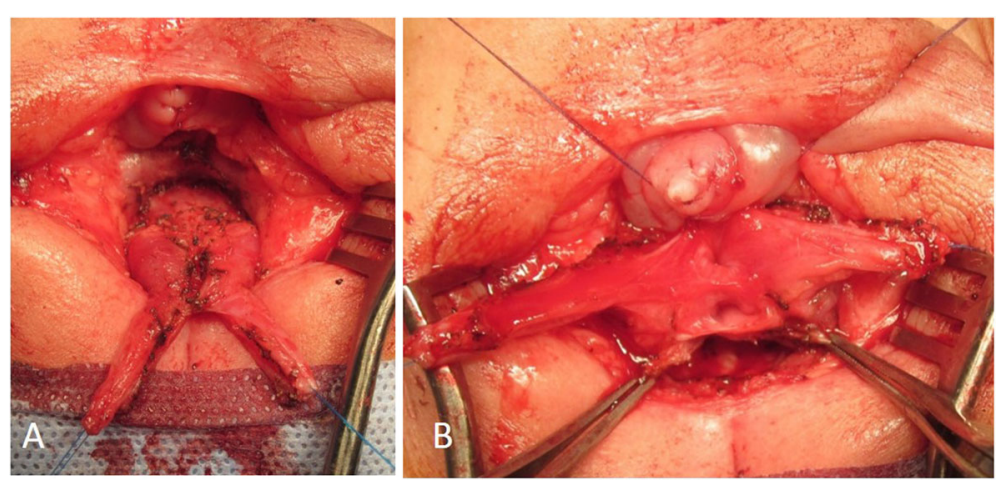

Fig. 7 Feminizing genitoplasty (cont.). A 10-month-old girl with CAH (same case in Fig. 5). The dissected urogenital sinus is split into two halves exposing both urethral and vaginal orifices 



Fig. 8 Feminizing genitoplasty (cont.). A 60-month-old girl with CAH. Another midline incision is made dorsally through the dissected urogenital sinus splitting it into two equal flaps that will be rotated and sutured to the mucosal collar on both sides encircling the glans (a-c). Note: the distal urogenital sinus is used to provide mucosal lining for the new vestibule. $\mathbf{d}$ Reconstruction of the labia minora on both sides of the vestibule

the second stage by detection of pain response (tachycardia) at the beginning of the procedure necessitating deeper anaesthesia and more analgesics.

In five cases (50\%), the technique of limited urogenital sinus mobilization adopted in this report succeeded in bringing the vaginal introitus down to the perineum. Those cases had originally a low vaginal take off from the urethra (type 1) [5]. Separate urethral and vaginal orifices could be easily identified upon labial retraction (Fig. 9 and 10). In the rest of cases, labial retraction showed a common but wide urogenital introitus perfectly lined by urogenital mucosa (Fig. 6). The outcomes have been considered satisfactory to a great extent for both parents and doctors regarding cosmesis and lack of functional complications (voiding problems). Longer follow up is still needed to assess the sexual function when these girls become sexually active.

\section{Discussion}

Genital surgery for CAH has passed several stages of development. Early reports described primitive procedures that comprised excision of the hypertrophied genital tubercle and simple cut-back vestibuloplasty [7]. Reduction clitoroplasty then replaced excision and rapidly became








Fig. 10 Delayed complementary stage 12 months after the primary operation (feminizing genitoplasty). a Typical feminine appearance after primary operation. $\mathbf{b}-\mathbf{d}$ Labial retraction revealed a well vascularized glans after reduction clitoroplasty; however, its larger than normal size was a major persistent concern for the parents that they requested further reduction to the size of the glans

the standard of care for these cases with special emphasis on preservation of its neurovascular supply [4]. Hendren and Crawford introduced the concept of vaginal pull-through to avoid cutting through the internal urinary sphincter when dealing with a high vagina [7]. The evolution of operative management continued with the efforts of imminent paediatric surgeons and urologists introducing new surgical approaches (Passirini flaps; partial/total urogenital sinus mobilization; laparoscopic and anterior sagittal trans rectal approaches) [8-12].

Early genital reconstruction may be recommended in cases of $\mathrm{CAH}$ to avoid the stigma that can affect these individuals with variable degrees through different cultures [4]. However, the separation and mobilization of a high vagina has remained challenging with less satisfactory surgical outcomes (often requires postoperative vaginal dilatations which is better avoided in childhood, in addition to redo surgeries after puberty) $[4,13]$. Therefore, the value of early vaginal reconstruction has been questioned in favour of delaying the whole repair after puberty. In this report, the author has adopted the third option in-between, which comprises early reconstruction of the external genitalia and delaying the more challenging vaginal reconstruction (if needed) to be performed after puberty [4]. By this, we can spare these young girls the unnecessary burden of possible complications of vaginal surgery during childhood; meanwhile, we also avoid the psychological problems that can result from keeping these girls to grow with virilized external genitalia.

In this report, we presented a detailed description of the surgical technique that has been applied in ten consecutive cases of $\mathrm{CAH}$. The technique has been fractionized into operative milestones clearly demonstrated by operative photos. The steps are perfectly reproducible with predictable outcomes. The outcomes have been considered satisfactory to a great extent for both parents and doctors achieving the goals of surgery according to the recommendations proposed at the latest DSD meeting [4]. Postoperative oedema was a common but transient complication. Wound disruption occurred in a single case that required delayed secondary suturing. To decrease the risk of wound disruption, it became the author preference to delay reduction of scrotalized labial skin (if needed) to be performed at a complementary stage 6-12 months later.

Reduction of the size of corporeal bodies of the virilized clitoris is an important goal of feminizing genitoplasty that should be achieved with preservation of neurovascular supply to the retained glans [13]. Control of bleeding by tourniquet application while performing this step allows for high precision during the dissection of the neurovascular bundle. In this study, the intact sensitivity and vascularity of the glans was specifically confirmed in three cases while performing a complementary second stage under general anaesthesia.

The separation and mobilization of the distal urogenital sinus together with the surrounding ventral erectile tissue accomplishes a second important goal for feminizing genitoplasty. The dissection continues up to the back of the lower end of the pubic symphysis without further extension into the upper retropubic space to avoid disturbing the normal anatomy of the bladder neck. A ventral 'lay-open' incision through the dissected urogenital sinus will perfectly expose the vaginal orifice in about $50 \%$ of cases (those with a low vagina; type 1) [5]. In cases with higher vaginal take-off from the urethra, the ventral incision will serve to widen the introitus and displace the erectile tissue laterally on both sides of new introitus [5].

The dissected distal urogenital sinus is split longitudinally into two equal flaps that are sutured to the mucosal collar encircling the glans. This step serves several functions: fixation of the urethra to the clitoris under the glans, providing urogenital mucosal lining for the new vestibule, and more effectively hiding the clitoris.

The technique adopted in this report (partial urogenital sinus mobilization) can be expected to achieve full correction of the anomaly (by bringing the vagina down to the perineum) in about $50 \%$ of cases. Although delayed vaginal surgery remains a valid option for the rest 
of cases with higher vagina [4], yet we believe that a residual uncorrected common urogenital sinus may not necessarily impair satisfactory penetrative intercourse in the future and that we may abort the surgical correction at that level. We present several observations that can support our assumption:

1. A common urogenital sinus is a normal anatomical finding in several species other than humans without causing functional problems. In humans, a normal vestibule may be considered a wide and shallow common urogenital sinus.

2. There are historical reports on cases with uncorrected common urogenital sinus who were engaged in sexual intercourse and got pregnant [14].

3. During contrast studies (genitograms) performed for girls with common urogenital sinus, not uncommonly the catheter passes preferentially into the vagina (indicating a high possibility for successful future penetrative vaginal intercourse through the common urogenital sinus). Similarly, in boys with severe hypospadias and a prostatic utricle, frequently, we experience difficulty in catheterizing the bladder as the catheter usually passes directly into the utricle.

4. The author has come to review a case report describing urethral intercourse among cases with Mullerian anomalies (Mayer Rokitansky Küster Hauser syndrome) [15]. Interestingly, there were several similar reports in the literature from different countries and different cultures $[15,16]$. More interestingly, many of these women unknowingly got engaged into urethral intercourse for years and were satisfied with their sexual life that they refused vaginal reconstruction when offered to them later [15-17].

5. Reports on sexual medicine distinguish between the internal genitals (vagina) having mainly a reproductive function and the role of external genitalia in female orgasm. Grafenberg described a role for the urethra in female orgasm representing a distinct 'erotogenic zone' in women on the anterior wall of the vagina [18].

6. The separation of the lower end of the vagina from the urethra as well as the extensive urogenital sinus mobilization are extremely challenging procedures and may be non-physiological and detrimental to this critical area. Even if possible, it does not seem to be always reproducible and can result in considerable scaring and fibrosis altering the sensitivity of this area (erotogenic zone). We also believe that these less satisfactory outcomes from the sexual point of view might have contributed to the developing campaign against early genital reconstruction.
On the contrary, some researchers reported on normal sexual outcomes in women with congenital adrenal hyperplasia who did not undergo any surgical correction [19].

In treating congenital anomalies, sometimes restoring the exact normal anatomy is infeasible or impractical. A typical example in paediatric surgery is the Ladd's procedure for treating intestinal malrotation; the success of operation depends on widening the base of mesentery by placing the intestine in an atypical position rather than trying to restore the normal anatomy. Contrarily, we believe that for years, the surgical correction of $\mathrm{CAH}$ has been more concentrating on restoring the anatomy rather than the function. Surgeons have been more concerned to create a visible separate vaginal orifice in the perineum via sophisticated flaps using a variety of tissues (not necessarily sensitive). Although these techniques have been shown to be feasible, many are not very well reproducible with less satisfactory outcomes; possible complications comprise variable degrees of scaring and fibrosis that can greatly affect function, cosmesis and sometimes continence $[13,20]$.

The feminizing genitoplasty described in this report is not different from the current techniques. What might be rather original in this report is the rationale for postponing/abandoning vaginal reconstruction during childhood (which is again controversial). The context of the above observations may draw some evidence to support our assumption (possibility of satisfactory sinus intercourse). This assumption (hypothesis) does not alter the current recommendations or surgical options [4] but rather supports choosing between them. The final judgement must be delayed until these patients grow and become sexually active. Meanwhile, this option needs to be discussed with different medical and may be nonmedical specialties who are concerned with this dilemma taking into consideration that we did not lose the chance of delayed vaginal reconstruction if turned to be necessary. The patients may even benefit from the developing science of tissue culture or other advanced vaginal reconstructive procedures in the future. Also, if the patient is well satisfied with her sexual life but there is fertility problem, this can be more simply managed by in vitro fertilization techniques which are becoming more popular among normal subjects.

\section{Conclusion}

In cases of congenital adrenal hyperplasia, reduction clitoroplasty combined with partial urogenital sinus mobilization can achieve predictable and satisfactory outcomes. In about $50 \%$ of cases, this approach is sufficient to bring the vagina down to the perineum. In the other half of cases with higher vagina, the possibility of 
satisfactory sinus intercourse can be studied when these girls grow and become sexually active; otherwise, a delayed vaginal reconstruction may turn to be necessary.

\section{Abbreviations}

$\mathrm{CAH}$ : Congenital adrenal hyperplasia; DSD: Disorders of sexual development; MRI: Magnetic resonance imaging; TUM: Total urogenital sinus mobilization; ASTRA: Anterior sagittal transrectal approaches

\section{Acknowledgements}

The author is deeply indebted to Dr. Supul Hennayake (Royal Manchester Children's Hospital) for his inspiring lecture on feminizing genitoplasty which he gave as an invited guest speaker at the 29th annual meeting of the Egyptian Pediatric Surgical Association held in Cairo in 2014.

\section{Author's contributions}

Single author. The author(s) read and approved the final manuscript.

\section{Funding}

None

\section{Availability of data and materials}

The datasets used and/or analysed during the current study are available from the corresponding author on reasonable request.

\section{Ethics approval and consent to participate}

Written consent was taken before operation in all cases. Owing to the retrospective nature of the study, an IRB number was not required, and the study was approved through expedited review by the scientific/ethical committee of the surgery department (Faculty of Medicine, Ain Shams University).

\section{Consent for publication}

Patient identity did not appear in any part of the manuscript; therefore, consent for publication was not required.

\section{Competing interests}

None

Received: 26 March 2020 Accepted: 1 September 2020

Published online: 20 October 2020

\section{References}

1. Lottman H, Thomas D. Disorders of sexual development. In: Thomas D, Duffy PG, Rickwood A, editors. Essentials of paediatric urology. London: Informa; 2008. p. 275-93.

2. Ganesan A, Smith GHH, Broome K, Steinberg A. Congenital adrenal hyperplasia: preliminary observations of the urethra in 9 cases. J Urol. 2002; 167:275-9.

3. Witchel SF. Congenital adrenal hyperplasia. J Pediatr Adolesc Gynecol. 2017; 30:520-34.

4. Mouriquand PDE, Gorduza DB, Gay CL, et al. Surgery in disorders of sex development (DSD) with a gender issue: if (why), when and how? J Pediatr Urol. 2016;12:139-49.

5. AbouZeid AA, Mohammad SA. Transformation of the female genitalia in congenital adrenal hyperplasia: MRI study. J Pediatr Surg. 2020;55:977-84.

6. AbouZeid AA, Mohammad SA. The 'Bifid bulb' sign to differentiate between high and low vagina in congenital adrenal hyperplasia. https://doi.org/10. 26044/ecr2020/C-05754.

7. Hendren WH, Crawford JD. Adrenogenital syndrome: the anatomy of the anomaly and its repair. Some new concepts. J Pediatr Surg. 1969:4:49.

8. Passerini-Glazel G. A new 1-stage procedure for clitoroplasty in severely masculinized female pseudohermaphrodites. J Urol. 1989;142:565 e8.

9. Ludwikowski B, Oesch Hayward I, Gonzalez R. Total urogenital sinus mobilization: expanded applications. BJU Int. 1999;83:820 e2.

10. Hamza AF, Soliman HA, Hay SA, et al. Total urogenital sinus mobilization in the repair of cloacal anomalies and congenital adrenal hyperplasia. J Pediatr Surg. 2001;36:1656-8.

11. Dòmini R, Rossi F, Ceccarelli PL, et al. Anterior sagittal transanorectal approach to the urogenital sinus in adrenogenital syndrome: preliminary report. J Pediatr Surg. 1997;32:714-6.
12. Rink RC, Metcalfe PD, Kaefer MA, Casale AJ, Meldrum KK, Cain MP. Partial urogenital mobilization: a limited proximal dissection. J Pediatr Urol. 2006;2: $351-6$.

13. Lee $\mathrm{P}$, Schober J, Nordenstrom A, et al. Review of recent outcome data of disorders of sex development (DSD): emphasis on surgical and sexual outcomes. J Pediatr Urol. 2012;8:611-5..

14. Pena A, Bischoff A: History of the treatment of anorectal malformations. In: Surgical treatment of Colorectal problems in children. Springer 2015; pp: 1-16.

15. El-Agwany AS. Asymptomatic uretheral intercourse associated with Mayer Rokitansky Küster Hauser syndrome type II: a case with crossed fused renal ectopia and scoliosis. African J Urol. 2017:23:48-51.

16. Rumley-Dawson E. A case of complete transverse septum of the vagina impeding delivery; urethral coitus. Lancet. 1900;156(4032):1646-7.

17. Ryckman J, Black A, Fleming N. Adolescent urethral coitus: 2 cases and review of the literature. J Pediatr Adolesc Gynecol. 2014;27:e9-e12.

18. Puppo V. Embryology and anatomy of the vulva: the female orgasm and women's sexual health. European Journal of Obstetrics \& Gynecology and Reproductive Biology. 2011;154:3-8

19. Crouch NS, Liao LM, Woodhouse CR, Conway GS, Creighton SM. Sexual function and genital sensitivity following feminizing genitoplasty for congenital adrenal hyperplasia. J Urol. 2008;179(2):634-8.

20. Oshiba A, Ashour K, Khairi A, et al. Evaluation of the early outcomes of partial urogenital sinus mobilization in females with congenital adrenal hyperplasia. Ann Pediatr Surg. 2020;16:13 https://doi.org/10.1186/s43159020-00024-0.

\section{Publisher's Note}

Springer Nature remains neutral with regard to jurisdictional claims in published maps and institutional affiliations.

\section{Submit your manuscript to a SpringerOpen ${ }^{\circ}$ journal and benefit from:}

- Convenient online submission

- Rigorous peer review

- Open access: articles freely available online

High visibility within the field

- Retaining the copyright to your article

Submit your next manuscript at $\boldsymbol{\nabla}$ springeropen.com 